\title{
DEBATES
}

\section{El capital social: revisión crítica de su uso y consecuencias en las ciencias sociales}

\author{
Social Capital: a critical review of it uses and consequences for \\ social sciences
}

\section{Cecilia Schneider \\ Micaela Diaz}

\section{Resumen}

Las múltiples formas de definir y utilizar el concepto de "capital social", nos invita a reflexionar sobre su naturaleza y efectividad teórica, desde que el famoso trabajo de Robert Putnam "Making Democracy Works" (1993) popularizó el término. Los problemas conceptuales y metodológicos de su aplicación surgen de la falta de distinción teórica entre los abordajes que le dieron origen. Por ello, es necesario en primer lugar identificar las tradiciones teóricas sobre las que se fundamenta, diferenciando sus tres principales representantes: Pierre Bourdieu, James Coleman y Robert Putnam. Como segunda tarea, nos centraremos en las críticas y preguntas que surgen del trabajo de Putnam. En tercer lugar, vemos cuáles son las consecuencias empíricas de esos postulados, en las investigaciones académicas y en los programas de intervención de organismos internacionales. Finalmente, este recorrido nos permite concluir que se trata de un concepto nuevo para etiquetar una vieja idea y que su actual uso acarrea las inconsistencias teóricas y ambigüedad desde su concepción.

\section{Palabras clave}

Capital Social; Revisión; Enfoques Teóricos; Consecuencias Empíricas.

\begin{abstract}
The many ways to define and use the concept of "social capital", since the famous work of Robert Putnam, "Making Democracy Works" (1993), popularized the term, invite us to review and reflect about its nature and theoretical effectiveness. The conceptual and methodological problems of implementation arise from the lack of theoretical distinction between the approaches that originated it. For this reason, first it is necessary to identify the theoretical traditions on which the concept is based, distinguishing its three main representatives: Pierre Bourdieu, James Coleman and Robert Putnam. Second, we focus on the critics and questions that arise from the work of Putnam. Third, we present the empirical consequences of these postulates, both in the academic research and in the intervention programs proposed by international organizations. Finally, this review allows us to conclude that this is a new concept to label an old idea, and its present use entails theoretical inconsistencies, vagueness and ambiguity, which has existed from the moment it was conceived.
\end{abstract}

\section{Keywords}

Social Capital; Review; Theoretical Approaches; Empirical Consequences. 


\section{Introducción ${ }^{1}$}

En los últimos años el término capital social ha ganado una extraordinaria y desmedida atención en las ciencias sociales. Fenómenos tan variados y disímiles como la participación ciudadana, los problemas de conducta juvenil, la socialización primaria y secundaria, la capacidad asociativa, el desempleo, el desarrollo económico, cuestiones de salud pública, la calidad de la democracia y el desempeño de las instituciones, entre muchos otros, han sido curiosamente explicados bajo esta misma lente (PORTES, 1998). La fabulosa proliferación de significados adjudicados al concepto revela que éste está transformándose en un término de moda (catch-call term) que explica toda gama de fenómenos.

El término fue introducido por G. Loury (1977), para referirse al conjunto de recursos inherentes a las relaciones familiares y a las organizaciones comunitarias que son útiles para el desarrollo cognitivo y social de las personas, especialmente, en su primera etapa de socialización. Posteriormente, Pierre Bourdieu (1986) realiza el primer análisis más sistemático del término. Su tratamiento es mas instrumental en tanto que le interesa comprender qué tipo de relaciones sociales favorecen o producen recursos necesarios a la acción colectiva y a la vez, cuál es la calidad de los mismos. Sin embargo, la definición más citada y a la que se le debe el posterior desarrollo es la de James Coleman (1988 y 1990) pese a que su vaguedad es aún mayor.

Como se sabe, quien mayor responsabilidad ha tenido en el uso del concepto ha sido Robert Putnam. Su obra se ha convertido, a su pesar o no, en un punto de referencia clave para instituciones como el Banco Mundial hasta de administraciones locales de diversas partes del mundo (por citar sólo aquellas ajenas al ámbito académico) en la búsqueda de soluciones a variados problemas. Pero, ¿cuál es el conjunto de acontecimientos que pretende capturar el concepto? ¿Qué es lo que intenta explicar? ¿Lo logra verdaderamente? ¿Cuáles son sus verdaderos aportes? ¿Se trata de un concepto novedoso? ¿Cómo se emparenta con otros conceptos acuñados desde las ciencias sociales?

\section{Sobre los origenes teóricos del capital social: tres tradiciones}

Según Bourdieu, el concepto de capital - en todas sus formas - es imprescindible para dar cuenta de la estructura y el funcionamiento del mundo social. En este sentido, la noción de capital no puede reducirse a su función instrumentalista que ha instalado la teoría económica clásica de maximización de

\footnotetext{
${ }^{1}$ Este artículo ha sido escrito en el marco del Proyecto Participación ciudadana local: contextos y culturas politicas en la Argentina actual, con subsidio PICTO104, UNDAV.
} 
utilidades y reducción de costos en las relaciones de intercambio de mercancías. Este tipo de intercambio (mercantil) constituye según Bourdieu, solo una forma dentro de las múltiples posibilidades de intercambio social que existen. En la diferenciación que hace de las formas de capital (haciendo énfasis en su campo de aplicación y en su potencial transformador), distingue entre el económico, cultural y social, donde este último aparece como un "capital de obligaciones y relaciones sociales" (BOURDIEU, 2001, p. 136).

Específicamente, lo define como "el conjunto de recursos actuales o potenciales vinculados a la posesión de una red durable de relaciones más o menos institucionalizadas de mutuo apoyo o reconocimiento o en otras palabras a la pertenencia a un grupo" (BOURDIEU, 1986, p. 248). El grupo provee a sus miembros con un capital de propiedad colectiva. Ese capital está representado por el tamaño de la red y por el volumen de capital (económico, simbólico o cultural) poseído por aquellos con quienes cada persona está conectada.

El capital social se basa entonces en relaciones de intercambio material y simbólico entre sujetos. En la construcción de estas redes, se combinan dos elementos. Uno es la intencionalidad - consciente o inconsciente - de los sujetos de establecer ciertas relaciones sociales y el otro es la potencialidad, en términos de beneficio que la pertenencia a éstas les supondrán y por lo cual se despliegan estrategias individuales o colectivas.

El fundamento teórico detrás de estos argumentos, se enmarcan en una doble perspectiva. La tradición estructuralista se evidencia en el énfasis que Bourdieu hace en las estructuras objetivas que orientan y coaccionan las prácticas sociales $y$, al mismo tiempo, el modelo constructivista se refleja en el carácter subjetivo de las estructuras mentales que condicionan y generan esas prácticas. "Se trata de un constructivismo en el que el análisis de las estructuras cognitivas es inseparable del análisis de las condiciones sociales en que aquellas tienen lugar" (GARCÍA INDA, 2001, p. 13). Así mismo, se puede identificar la influencia del pensamiento Durkheimiano a partir del énfasis que Bourdieu hace de cuestiones como los "ritos de institucionalización" (necesarias para producir y reproducir conexiones útiles y duraderas); las relaciones casuales de cualquier tipo (que generan obligaciones duraderas sustentadas en sentimientos subjetivos como la amistad o en garantías institucionales); el reconocimiento mutuo entre los miembros de un grupo; las formas "más o menos institucionalizadas de delegación" que existen en un grupo (necesario para producir y reproducir las conexiones o relaciones útiles y duraderas).

Coleman por su parte, define capital social por su función: se trata de un aspecto de la estructura social que facilita ciertas acciones de los actores que están 
dentro de ella. En su comparación con otros tipos de capital, el social es menos tangible y no completamente fungible. La similitud más evidente entre ambos abordajes, es que los dos consideran capital social como un recurso. Para los dos autores, el concepto representa el conjunto de recursos ganados, obtenidos o a obtener a través de relaciones sociales, pese que para Coleman este recurso tiene un carácter individual y para Bourdieu fundamentalmente colectivo.

Según Coleman, "el capital social se define por su función. No es una sola entidad, sino una variedad de diferentes entidades, con dos elementos en común: todos ellos consisten en algún aspecto de las estructuras sociales, y facilita ciertas acciones de los actores, ya sean personas o actores corporativos, dentro de la estructura" (COLEMAN, 1988, p. 98) ${ }^{2}$. Además, "es un recurso importante para las personas que puede afectar en gran medida su capacidad de actuar y su calidad de vida" (1988, p. 118). Identifica tres formas de capital social: obligaciones y expectativas (que dependen de la confianza y surge en contextos de intercambio); el potencial de información inherente a las relaciones sociales (donde se consideran los beneficios que pueden obtener los actores para alcanzar sus fines a partir de la información que poseen los otros sujetos); y las normas sociales y sanciones efectivas (que cohíben realizar ciertas acciones y estimulan llevar a delante otras). Así, al entender el capital social a través de las normas sociales, los canales de información, expectativas sociales y las obligaciones de los individuos dentro de una estructura social específica, Coleman lo identifica como un recurso disponible y controlable por los sujetos: "Si comenzamos una teoría de la acción racional, en la que cada actor tiene control sobre ciertos recursos e intereses en ciertos recursos y eventos, el capital social constituye un tipo particular de los recursos disponibles" (1988, p. 98). Esto marca otra diferencia con Bourdieu, donde los recursos están insertos en la estructura social.

Macedo Castillejos (2009) también diferencia entre el carácter acumulativo e histórico del capital social en Bourdieu, que implica la acumulación de las relaciones vinculadas y conectadas a redes sociales, en contraste con la naturaleza productiva o funcional de dichas relaciones que presenta Coleman. Además, sitúa al modelo de Coleman cercano al constructivismo al resaltar el rol preponderante que tienen las decisiones de los actores sociales - donde el actor individual tiene poder de elecciónpara dar forma al mundo social.

Otra diferencia entre ambas perspectivas advertida por Tzanakis (2013), es que para Coleman el capital social adquiere la naturaleza de un bien público, donde

\footnotetext{
${ }^{2}$ Todas las citas de J. Coleman son de traducción propia.
} 
las contribuciones directas de los actores, beneficiarán al conjunto. Por el contrario, para Bourdieu el capital social reproduce la desigualdad social pero a la vez puede incrementar la integración dentro de grupos específicos. Así, según Tzanakis, ambos autores tienen una perspectiva pesimista de la sociedad pero fundamentada en diferentes razones. Para Bourdieu, porque entiende al capital social como un recurso escaso y como un mecanismo de reproducción de clase que perpetua la desigualdad estructural. Coleman por su parte, lo entiende como un potencial bien público aunque resalta la cada vez mayor debilidad de los lazos sociales de la comunidad (debido a que las redes son cada vez más abiertas, lo que conlleva a establecer relaciones menos densas y menos unidas). Como resultado, el potencial del capital social para convertirse en un bien público compartido, se encuentra limitado. Coleman encuentra la solución para superar el problema del suministro de estos bienes públicos en "la sustitución de algún tipo de organización formal de la organización social voluntaria y espontánea que en el pasado ha sido la principal fuente de capital social" (1988, p. 118). Al igual que en el caso del sociólogo francés, aquí también es posible identificar las raíces Durkheimianas de este razonamiento.

En sintonía con estas tradiciones, otras definiciones han condensado elementos de ambas perspectivas. En el caso de Lin (2001), considera al capital social como recursos insertos en la estructura social y arraigado a redes (a los cuales los sujetos pueden acceder y movilizar de manera intencional a partir de lazos sociales). De este modo, el autor fusiona elementos de Coleman como de Bourdieu: hace énfasis en el manejo instrumental del capital social y prioriza la perspectiva del rational choice donde "la acción es la que lleva a la conformación de la estructura social mediante la movilización de los recursos sociales o del capital social" (LIN, 2001, p. 53) pero también como Bourdieu, enfatiza la intencionalidad y potencialidad de las relaciones de intercambio en términos del beneficio que la pertenencia a las redes les supondrá.

Pero sin duda fue el conocido trabajo de Putnam (1993) el que inició un largo camino en el uso y abuso de la expresión. Preocupado por establecer las causas que determinan la existencia o no de gobiernos eficaces Putnam analiza por qué en Italia el rendimiento institucional de los gobiernos regionales del norte es significativamente mejor que en el sur del país. En el norte italiano, donde los ciudadanos participan activamente en clubes deportivos, asociaciones literarias y sociedades corales, los gobiernos son "eficientes en su funcionamiento interno, creativos en sus iniciativas políticas, y efectivos en la ejecución de estas iniciativas" (1993, p. 81). En el sur de Italia, en cambio, donde las pautas de compromiso cívico son muy débiles sus gobiernos son corruptos e ineficientes. Según concluye, el capital 
social consiste en "el conjunto de redes sociales y vínculos de reciprocidad y confianza que se generan entre los miembros de una comunidad en el marco de la cooperación social y la interacción" (1993, p. 221). Cuanto más estables son esas pautas de compromiso cívico más rica es esa sociedad en términos de capital social. El sencillo argumento que sostiene el autor indica entonces que la tradición asociativa y el compromiso cívico de los ciudadanos afectan diferencialmente el desempeño político institucional, es decir, el modo y el rendimiento de los gobiernos en las regiones del norte y del sur de Italia. De este modo los italianos del sur tendrían los gobiernos más ineficaces porque su capacidad para construir lazos horizontales, asociarse y confiar en sus pares, emprender iniciativas de ayuda mutua es comparativamente paupérrima a los italianos del norte.

En los esfuerzos de Putnam por relacionar la dinámica asociativa y el compromiso cívico con el desempeño de las instituciones políticas, es posible identificar una vinculación conceptual entre el enfoque tradicional de la cultura política y los enfoques institucionalistas popularizados en los años 90 (JORDANA, 2000). Veremos más adelante que esta conexión, trasciende el ámbito académico y es aplicada actualmente desde distintos organismos internacionales como el Banco Mundial o el PNUD. Al identificar Putnam una sociedad civil fuerte, con altos niveles de "compromiso cívico", sugiere una correspondencia entre las características estructurales de la sociedad y un cierto tipo de cultura política o "cívica". Según Edwards y Foley (2001), este enfoque de la cultura cívica (fuertemente difundida en la ciencia política norteamericana), destaca el carácter socializador de las asociaciones y resalta el papel crucial que tienen factores como la existencia de ciertos compromisos morales entre los ciudadanos en el desempeño de la democracia. Elementos como la confianza - entre los individuos, en el gobierno y en los funcionarios públicos -, la tolerancia y el optimismo son vistos como componentes integrales de capital social directamente vinculados a su impacto beneficioso en la participación y el compromiso cívico y la democracia en general. En el trabajo de Gabriel Almond y Sidney Verba (1963), se encuentra el argumento de que la cultura política de una sociedad podía convertirse en un factor explicativo de la estabilidad y de la modernización democrática. Entre las conclusiones de su trabajo, destacan la existencia de un tipo de cultura política - al que llaman cívica - como la más compatible con el sistema democrático, a la que caracterizan como la combinación entre las orientaciones activas y pasivas de los ciudadanos; entre las dosis de consenso y disenso y finalmente, entre los niveles de confianza social e interpersonal y de relaciones afectivas e instrumentales con la política (SCHNEIDER Y AVENBURG, 2015). Así, este abordaje también destaca la confianza como ingrediente clave, en este 
caso, para la estabilidad democrática. En el trabajo de Putnam se retoman muchos de estos elementos, pero con el interés de argumentar y demostrar que la vida asociativa tiene un impacto relevante en la cultura política y en el compromiso cívico y no al revés como lo plantean Almond y Verba.

\section{Sobre las criticas: consecuencias empiricas del concepto}

La revisión de las tres tradiciones nos permite identificar las diferentes definiciones del concepto capital social, haciendo énfasis en sus puntos de encuentro y sus divergencias. También reflejan las tradiciones teóricas sobre las cuales cada uno desarrolló sus argumentos. Sin duda, se trata de un concepto que encierra múltiples dificultades, no solo por su ambigüedad y vaguedad, sino también por las consecuencias empíricas que estos postulados tienen.

Haciendo hincapié en el trabajo de Putnam, la primera dificultad que observamos es que no le adjudica un papel decisivo en la explicación a la dispar influencia del desarrollo económico en las regiones del norte y sur de Italia, así como sus diferentes tradiciones históricas. El mismo advierte que en las regiones donde ha imperado regímenes republicanos y formas de gobierno horizontales o poco jerarquizadas durante la Edad Media se desarrolló una tradición asociativa y un sistema de gobierno que ha conducido a tener gobiernos regionales más satisfactorios. Por el contrario, en el sur italiano, han prevalecido administraciones absolutistas y relaciones de corte más jerárquicas. Aun aceptando esta relación convendría preguntarse, entonces, ¿̨uál de los dos aspectos - tipo de régimen político o tipo de comunidad cívica - tiene mayor importancia? Así planteadas las cosas no sería difícil encontrar ejemplos de sistemas absolutistas tremendamente eficaces. Pese al atractivo teórico que puede tener el argumento esgrimido por Putnam, lo cierto es que no se comprende el modo en que las observaciones empíricas del concepto definidas por el autor afectan el buen desempeño de las instituciones y la democracia en términos más generales - relación que por otra parte y tal como señala el mismo es su principal preocupación teórica.

Pese al entusiasmo de los culturalistas, Putnam no logra describir el mecanismo mediante el cual las tradiciones cívicas impactan en el quehacer diario de los funcionarios que componen cualquier gobierno o institución burocrática. ¿Por qué no logra esto? En gran medida porque el concepto adolece de una tremenda debilidad en su definición teórica y empírica. ¿Qué fenómeno captura el término capital social? Al responder esta pregunta los problemas surgen en forma considerable y sus supuestos atractivos se desdibujan. Como ya se señaló, en los últimos años numerosos estudios han intentado curiosamente más allá de toda discusión 
conceptual definir los indicadores más adecuados para medir el nivel existente de capital social en cualquier sociedad (SUDARSKY, 1999). En esas distintas definiciones y usos la operacionalización ha implicado una lista de categorías de lo más variada: por ejemplo, pertenencia a asociaciones de todo tipo (clubes de fútbol, grupos corales, fundaciones económicas, asociaciones de vecinos, partidos políticos); vínculos informales y formales de distinta intensidad; redes y normas de confianza personal, predisposición a entablar relaciones de confianza, relaciones de cooperación colectiva etc.

Observamos que la aparente complejidad y multidimensionalidad se reduce al uso de un solo indicador al que Putnam llama "sociabilidad o comunidad cívica" compuesto por las siguientes categorías: densidad de la vida asociativa (es decir, cantidad de asociaciones por habitante), regularidad en la asistencia a votaciones generales, asistencia a referéndum y, por último, lectura y suscripción de periódicos. A su vez estos cuatro indicadores combinados conforman un índice que mide el stock disponible de capital social de un individuo, grupo o comunidad. Al construir un índice se asume que sus componentes pueden formar una medida total, general y única de capital social; lo cierto es que este intento encubre distinciones conceptuales relevantes (PORTES, 1998; FISHMAN, 2000). Por ejemplo, aquellas que refieren a los diferentes objetos y fines que pueden perseguir las asociaciones tan distintas como Greenpeace o la que nuclea a los amantes de la navegación de barquitos caseros en la Plaza Francia de Buenos Aires.

Putnam señala que "el buen gobierno en Italia es un subproducto de los grupos musicales y de los clubes de fútbol” (1993, p. 176). Ironizando sobre la propia afirmación del autor no se entiende, entonces, por qué el sur italiano no ha contado con "buenos gobiernos". Sin embargo la relación entre participación en asociaciones y la calidad de vida política está lejos de ser axiomática. Las organizaciones pueden orientarse hacia el establecimiento de beneficios exclusivos para sus propios miembros sin que los efectos de esta cooperación trasciendan sus fronteras. En este mismo sentido, no alcanza con saber cuánta gente participa en organizaciones civiles como hace el autor si no dedicamos el mismo esfuerzo por distinguir los tipos de vínculos que esa vida asociativa genera o puede generar. Mucho tiempo antes la sociología clásica de la mano de Durkheim había establecido la diferente capacidad que tienen los grupos débilmente integrados respecto de los fuertes para generar vínculos sociales de más largo aliento.

El autor clasifica a los grupos en función de la horizontalidad y la verticalidad de sus relaciones. Pero, otra vez, ¿la interacción social per se basta? ¿No debiéramos preguntarnos acerca de las normas que siguen los miembros de estas distintas 
organizaciones, o cuán democráticos son sus objetivos y maneras de organizarse? En todo caso, el uso del capital social generado no depende sólo de la finalidad y otras características del grupo; depende también de la extensión de la actividad asociativa: ¿cuál es el puente entre los aficionados, por ejemplo, al futbol o al canto coral y la vida pública/política? $\mathrm{O}$, en términos más precisos, ¿¿de qué manera sus intereses, las pautas de cooperación que funcionan en el interior del grupo y las relaciones de confianza generadas se vinculan con el buen o mal rendimiento de las instituciones políticas? ¿Cuáles son esas microconexiones?

Ignorar estas cuestiones ha provocado que buena parte de la literatura e investigación en el tema subraye únicamente los efectos positivos del capital social a través de su rol en el control social, en la constitución de redes extrafamiliares o en la calidad de la vida política (BOIX y POSNER, 2000; LEVI, 1996). Pero las consecuencias negativas del mismo proceso permanecen en la oscuridad: las mafias también crean capital social o el funcionamiento de los carteles colombianos que basan su funcionamiento en redes de confianza y cooperación entre sus miembros (RUBIO, 1997). Este tipo de organizaciones criminales son generadoras de capital social, aunque sus efectos en términos sociales sean negativos.

Otra distinción especialmente importante refiere a la relación entre la confianza y la pertenencia asociativa. La lógica de la argumentación seńala que la participación en asociaciones con fines públicos genera normas y relaciones de confianza. Esta confianza es a su vez uno de los motores del capital social porque establece expectativas legítimas de cooperación entre los miembros. Aun dejando de lado aquí la probable circularidad del argumento, en el trabajo de Hall (1999) sobre la existencia y desarrollo de capital social en G. Bretańa, se observa que éste en tanto medida general permanece alto fundado en una alta capacidad de los ingleses para conformar asociaciones de diverso tipo mientras que la confianza social vive un constante declive desde hace décadas. Por lo visto, ambos componentes del capital social no necesitan variar conjuntamente y si no lo hacen, ¿es posible concluir que el capital social está en alza en dicho país mientras uno de sus componentes sufre una disminución significativa y sostenida a través del tiempo? ¿Qué pesa más las actitudes de confianza o las relaciones sociales? Por otra parte la compleja relación entre el sentimiento de confianza, las normas que lo promueven y algún tipo de relación social debería ser estudiada y no dada por cierta como hace en ocasiones la literatura sobre el tema.

Otra de las críticas en relación a la confianza como eje articulador del capital social es que al presentarlo Putnam como "recurso moral" (PUTNAM, 1993), que supondría el reconocimiento del otro más allá de la interacción entre quienes 
comparten una afinidad electiva - ya sea religiosa, deportiva o recreativa. "Si no doy este paso, el "capital social - construido sobre la base de la confianza interpersonal acotada - es parcial, edifica una comunidad que puede ser excluyente" (DAGNINO, OLVERA y PANFICHI, 2006, p. 30). La cultura del racismo en Estados Unidos o la discriminación hacia comunidades indígenas en América Latina, demuestran esto.

\section{Sobre su uso y limitaciones metodológicas}

En concordancia con la falta de consistencia teórica y la ambigüedad del concepto, su uso empírico también es de lo más heterogéneo - no solo en los campos de aplicación sino también en los indicadores utilizados para mediar los niveles existentes de capital social. La inexistencia de un marco teórico consistente del capital social, se traduce en problemas metodológicos para cuantificar empíricamente el fenómeno. Veamos algunos ejemplos en los proyectos emprendidos por organismos internacionales desde hace décadas muy proclives a la utilización del concepto y a la asunción a priori de la vinculación entre la existencia de formas de capital social y el desarrollo económico sostenible. Fine (1999), advierte que el Banco Mundial retoma de Putnam el argumento que posiciona al capital social como un prerrequisito para una efectiva política pública y como un elemento que trabaja a través y dentro de los estados y los mercados, basado en el rol de factores no económicos (confianza, cultura, redes, participación, etc.) para el desarrollo económico. Según este autor, priorizar los factores no-económicos e interpretar al capital como una relación social en lugar de pensarlo como un "recurso atomizado", le ha permitido al organismo evitar incluir en la discusión factores como las relaciones de clase y de poder.

Por su parte, el Banco Mundial elabora su propia definición:

El capital social se refiere a las instituciones, relaciones y normas que conforman la calidad y cantidad de las interacciones sociales de una sociedad. Numerosos estudios demuestran que la cohesión social es un factor crítico para que las sociedades prosperen económicamente y para que el desarrollo sea sostenible. El capital social no es sólo la suma de las instituciones que configuran una sociedad, sino que es asimismo la materia que las mantiene juntas (BANCO MUNDIAL, 2015, s/p).

Este organismo identifica cinco dimensiones a partir de las cuales mide el capital social: grupos y redes (basados en el argumento de que el compromiso de las personas para organizarse y movilizar recursos en pos de un interés común generan mayores niveles de capital social); confianza y solidaridad (incluye elementos informales y subjetivos que intervienen en las actitudes y en las formas en que las 
personas interactúan unas con otras. Se basa en el argumento que mayores niveles de confianza entre personas e instituciones, facilitan la realización de acuerdos y transacciones); acción colectiva (se utiliza y se mide como un indicador indirecto del capital social); cohesión e inclusión social (hace referencia a la resolución conjunta de conflictos y necesidades a partir del trabajo conjunto y la participación, en pos de alcanzar intereses comunes); información y comunicación (asume que el mantenimiento y la mejora del capital social dependen de la capacidad de los miembros de una comunidad para comunicarse entre sí con otras comunidades) ${ }^{3}$.

En los trabajos académicos también se refleja la falta de consenso sobre la definición de capital social y como se acarrean también las inconsistencias teóricas que se originaron desde su concepción. Adicionalmente, no siempre es posible distinguir con claridad dentro de que perspectiva se enmarcan estos estudios o si se refieren a las ideas de uno u otro autor.

Dentro de aquellos que adoptan el modelo de Putnam, parten de asumir una correlación positiva entre las formas de capital social y desarrollo eficiente de instituciones - de múltiples tipos - sin explicar teóricamente como se vinculan; o no hacen referencia a las condiciones que son necesarias para que esta correlación ocurra; se sobredimensiona el factor cultural para explicar fenómenos políticos, por mencionar solo algunas de las dificultades. Los inconvenientes que se detectan en Putnam a partir del método estadístico ${ }^{4}$ que utiliza para comprobar sus afirmaciones

\footnotetext{
${ }^{3}$ A modo de ejemplo, aquí se mencionan algunos de los indicadores presentes en cada dimensión, que incluyen mediciones sobre: Grupo y redes: membresía, diversidad de los miembros, la extensión de funcionamiento democrático, la extensión de las conexiones con otros grupos. Confianza: refiere a relaciones establecidas y casuales (incluye mediciones basadas en las expectativas de comportamiento o un sentido de normas compartidas) así como también con las instituciones públicas (incluye mediciones sobre la equidad de las reglas, procedimientos oficiales, solución de controversias y la asignación de recursos). Acción colectiva: actividades de voluntariado, finalidad de la participación en actividades comunitarias, frecuencia, demandas, etc. Cohesión social: Quiénes están incluidos en la acción colectiva, la toma de decisiones y el acceso a los servicios. Esto incluye mediciones sobre la percepción de la unidad social y la convivencia así como también experiencias concretas de exclusión de los procesos de toma de decisiones o de ciertos servicios. Información y comunicación: disponibilidad de medios de comunicación y fuentes de información (oficina de correos, teléfono, prensa, radio y televisión, etc.). Para acceder a las dimensiones y la operacionalización de los indicadores, ver el Cuestionario Integrado para la Medición del Capital Social elaborado por el Banco Mundial (WORLD BANK, 2015).

${ }^{4}$ Dentro de la crítica más destacada en relación al método, Jackman y Miller (1996) indican que al desagregar los indicadores que Putnam utiliza para probar la relación entre las variables cultura cívica y desempeño político, encuentran que no hay una relación significativa estadística entre ambas. Otras
} 
y generalizaciones, también se reflejan en las investigaciones que se esfuerzan por medir el capital social. En este sentido, las investigaciones que son más afines con la perspectiva teórica de Bourdieu - donde se parte de una visión no individualista (específicamente a partir de redes o grupos) y cultural del capital social - son las que según Edwards y Foley (2001), permite comprender con claridad y coherencia como podría medirse y "pesar" el capital social, aspecto que no se encuentra en los trabajos de Coleman y Putnam. Lo que identifican en Bourdieu, es una definición específica al respecto:

El volumen de capital social poseído por un individuo dependerá tanto de la extensión de la red de conexiones que éste pueda efectivamente movilizar, como del volumen de capital (económico, cultural o simbólico) poseído por aquellos con quienes está relacionado (BOURDIEU, 2001, p. 150).

Quienes, por el contrario, optan por el modelo de Coleman recaen en el problema teórico-metodológico de partir de aspectos micro (como pueden ser ciertas pautas de comportamiento individual o las características de las relaciones familiares) con fenómenos macro (como ser el desempeño institucional o el incremento de las tasas de criminalidad en una comunidad). La influencia de la perspectiva de Coleman se puede ver en variados estudios empíricos, que intentan demostrar el efecto que tienen agentes del entorno micro-social como la familia, en el desarrollo y el alcance de logros individuales. Esto es interpretado en términos de posesión individual de capital social. Así por ejemplo, se pretende relacionar la deserción escolar, la delincuencia o el aumento de las tasas de criminalidad con factores que producen capital social positivo o negativo como ser la procedencia étnica de la familia o la cantidad de horas que los hijos miran la televisión. Como advierte Fine (1999), este tipo de estudios parten de especular sobre la existencia de ciertas relaciones causales que luego intentan validar estadísticamente. Realizan un salto del nivel individual al social a partir del uso de indicadores macro-estructurales y estadísticas evitando cuestionarse sobre los argumentos teóricos que vinculan las variables de análisis así como los mecanismos y procesos que intervienen en la reproducción social ${ }^{5}$. Análisis más recientes sobre el uso académico del concepto capital social, advierten sin embargo que las investigaciones que se basan en aspectos cualitativos del capital social

críticas refieren a la debilidad de los indicadores utilizados por Putnam, especialmente para medir el grado de "civilidad" a través del tiempo.

${ }^{5}$ Para ver diversos estudios cuantitativos que se enmarcan en esta lógica, consultar Fine (1999). 
utilizan como referencia la perspectiva de Coleman mientras estudios cuantitativos de gran escala se basan en la perspectiva de Putnam, aunque hacen uso instrumental del concepto (priorizando el modelo metodológico de Putnam y no su marco conceptual) (MACEDO CASTILLEJOS, 2009) ${ }^{6}$.

Quienes han revisado las producciones académicas que se enmarcan dentro de la concepción del capital social (FINE, 1999; MACEDO CASTILLEJOS, 2009; TZANAKIS, 2013; BJØRNSKOV y SØNDERSKOV, 2013), advierten que no existe en la actualidad un uso y entendimiento compartido del concepto y también que, si bien las herramientas analíticas utilizadas para la investigación empírica se han complejizado y mejorado, los referentes teóricos dentro de los cuales están investigaciones se enmarcan siguen siendo diversos y ambiguos. Bjørnskov y Sønderskov (2013) analizan la consistencia lógica y la coherencia interna del término capital a partir de evaluar ocho criterios (familiaridad; resonancia; parsimonia; coherencia; diferenciación; profundidad; utilidad teórica y utilidad de campo) ${ }^{7}$. Este análisis les permite concluir que no se trata de un buen concepto en las ciencias sociales. Sostienen que la definición de Putnam, cumple con los tres primeros criterios y que este es el principal motivo por el cual el concepto ganó una inmensa popularidad rápidamente. Estos son: familiaridad (el término permite asociarlo intuitivamente a su definición como factor de producción que se basa en las relaciones sociales. Dicho de otro modo, el concepto se etiqueta bajo un nombre que tiene sentido y que describe su contenido); además tiene resonancia (es fácil de recordar especialmente porque nos remite o nos permite identificar elementos de nuestra cotidianidad) y parsimonia (que refiere a la longitud de la definición. Según los autores, la de Putnam es una definición corta y precisa que caracteriza el concepto sin nombrar numerosos atributos y por ello es más fácil de recordar y aplicar característica que a la vez perjudica su diferenciación respecto de otros conceptos). Sin embargo, los criterios que identifican como negativos son los que más influyen en la eficacia de un concepto. En este sentido, lo destacan como: internamente incoherente (al definirlo como todos los aspectos de la organización social que mejora la cooperación, el concepto se torna inadecuado en el análisis empírico para establecer tanto las causas como los efectos del capital social); poco diferenciado (la amplitud del concepto, siendo que el capital social puede ser casi cualquier cosa que genere

\footnotetext{
${ }^{6}$ En revisión sistemática de trabajos académicos que realiza este autor, identifica que es en el área de salud y medicina, donde más se adopta la perspectiva y metodología de Putnam.

${ }^{7}$ Basan el análisis retomando el modelo propuesto por Gerring (1999) que para evaluar la bondad (o no) de los conceptos en las ciencias sociales. Para una descripción en detalle de cada criterio, ver Bjørnskov y Sønderskov (2013).
} 
resultados socialmente ventajosos, permite superponerlo con otros conceptos cercanos. Esto se refleja en las más diversas maneras en las que el concepto de operacionaliza, obteniendo resultados empíricos poco robustos y poco fiables); falla en el criterio de utilidad campo (la mayoría de los elementos que los constituyen ya existen); profundidad (si bien puede considerarse un concepto profundo ya que se utiliza para explicar un gran número de fenómenos y por lo tanto a él se asocian varios resultados, esto responde principalmente a la naturaleza indiferenciada del concepto capital social que permite que diferentes fenómenos puedan ser cobijados bajo esta perspectiva); si bien no puede negarse su utilidad teórica - por la cantidad de disciplinas que de él hacen uso para abordar sus estudios. Sin embargo, es necesario cuestionarnos si verdaderamente el concepto ayuda a formular nuevos postulados o a reformular los existentes.

En síntesis, podemos apreciar que lejos de adoptar un marco argumentativo específico vinculado a algunas de las perspectivas teóricas analizadas, el abordaje académico multidisciplinario reconstruyen un concepto de capital social absolutamente sui generis, que abarca cada vez más fenómenos y pierde cada vez más precisión y capacidad explicativa, transformándose en concepto de alto espectro para las ciencias sociales.

\section{Conclusiones}

Lejos de caer en desuso debido a su ambigüedad y vaguedad, el capital social sigue siendo el enfoque sobre el que se enmarcan la más variada gama de investigaciones académicas y sobre el que se fundamentan programas de intervención, focalizados en la reducción de la pobreza y el fomento del desarrollo. ¿A qué responde esto? ¿Se trata de una incorrecta interpretación del término o por el contrario, de un concepto que arrastra sesgos e inconsistencias desde su propia concepción? A lo largo de este trabajo, intentamos mostrar que la respuesta es afirmativa para ambos cuestionamientos. Nos enfrentamos así con una doble dificultad: por un lado, con un concepto que requiere ser criticado por su inconsistencia teórica. Por otro, con múltiples interpretaciones, valoraciones, usos y aplicaciones que no tienen en cuenta las limitaciones que el concepto acarrea.

Una primera tarea, fue diferenciar las definiciones en sus tres versiones más conocidas, la de Bourdieu, Coleman y Putnam. Además de rastrear las perspectivas y tradiciones teóricas detrás de cada uno de ellos. A modo de resumen, podemos diferenciar los fundamentos micro-económicos de base durkheimiana sobre los cuales Bourdieu construye su noción de capital social de la incorporación de elementos proveniente de la teoría de la elección racional identificable en Coleman. Pero 
también, Edwards y Foley, señalan diferentes influencias teóricas si se los observa de una perspectiva macro-sociológica: mientras Bourdieu es heredero de la tradición estructuralista de influencia marxista, Coleman se mantiene dentro de la tradición funcionalista de Durkheim y Parsons. Si en cambio nos centramos en la definición de capital social de Putnam, es posible identificar elementos weberianos presentes también en el enfoque tradicional de la cultura política (EDWARDS y FOLEY, 2001). Siguiendo la diferenciación entre las definiciones funcionalistas del capital social y aquellas que no lo definen por sus efectos que hace Portes (2000), en Putnam se refleja este característica funcionalista del término donde el capital social es cada aspecto de las organizaciones sociales que mejoran la eficiencia de la sociedad ${ }^{8}$.

A modo de conclusión cabe preguntarnos si no se trata de un nuevo término para una vieja idea. Pese a la popularidad del término, éste no encarna una idea absolutamente nueva y mucho menos original en la teoría sociológica. La pregunta por el lazo social y el interés en las relaciones de tipo comunitarias ha estado presente, con cierta perseverancia, en la historia del pensamiento sociológico clásico. Como es sabido, fue Emile Durkheim (1987) quien trabajó extensamente el concepto de solidaridad y las formas de la interacción social en las sociedades antiguas y modernas. En síntesis, el enfoque durkheimiano ha concebido a la vida asociativa bajo la forma de una gran malla de contención social proveedora de sentidos a la vida individual, una forma de pertenencia capaces de ligar a las personas a partir de intereses comunes $y$, finalmente, comprende la organización social como una consecuencia de las relaciones de solidaridad.

Parte del atractivo que tiene la noción de capital social reside en que vuelve a capturar aportes clásicos ya realizados por Simmel (1986) y por E. Durkheim mucho tiempo atrás. Pero a diferencia de éstos, la moda del capital social deja abiertas más preguntas de las que intenta responder, y cuando lo hace aporta claroscuros.

Cecilia Schneider é Professora e Pesquisadora da Universidade Nacional de Avellaneda da Argentina. E-mail: cecilia.schneider1@gmail.com.

Micaela Diaz é Doutoranda da Universidad de St. Gallen na Suiça. E-mail: mdiazrosaenz@yahoo.com.ar.

\footnotetext{
${ }^{8}$ Bjørnskov y Sønderskov (2013), advierten sobre la posterior definición de Putnam en su obra "Bowling Alone" (2000) no tiene este rasgo funcionalista, definiéndose el capital social sólo por su contenido.
} 


\section{Referências}

ALMOND, Gabriel; SIDNEY, Verba. La Cultura cívica: Estudio sobre la participación política democrática en cinco naciones. Madrid: Euramérica, 1963.

BANCO MUNDIAL. ¿Que es el Capital Social? Disponível em: <http://web.worldbank.org/WBSITE/EXTERNAL/TOPICS/EXTSOCIALDEVELOPMENT/EX TTSOCIALCAPITAL/0,,contentMDK:20187568 menuPK:410388 pagePK:148956 piPK:21661 8 -theSitePK:401015,00.html? iframe=true\&width $=80 \% \&$ height $=80 \%>$. Acesso em: 5 ago. 2015.

BJØRNSKOV, Christian; SØNDERSKOV, Kim. Is social capital a good concept? Social Indicators Research, v. 114, n. 3, p. 1225-1242, 2013.

BOIX, Carles; POSNER, Daniel. Capital social y democracia. Revista Española de Ciencia Política, n. 2, p. 159-186, 2000.

BOURDIEU, Pierre. The Forms of Capital. In: RICHARDSON, John G. (Ed.). Handbook of Theory and Research for the Sociology of Education. Nueva York: Greenwood Publishing Group, 1986. p. 241-258.

. Las formas del capital. Capital económico, capital cultural y capital social. In: BOURDIEU, Pierre. Poder, derecho y clases sociales. Bilbao: Desclée de Brouwer, 2001. p. 131-164.

COLEMAN, James. Social capital in the creation of human capital. American Journal of Sociology, v. 94, p. 95-120, 1988.

. Foundations of Social Theory. Cambridge: Belknap Press, 1990.

DAGNINO, Evelina; OLVERA, Alberto; PANFICHI, Aldo. Introducción: Para otra lectura de la disputa por la construcción democrática en América Latina. In: DAGNINO, Evelina; OLVERA, Alberto; PANFICHI, Aldo (Coord.). La disputa por la construcción democrática en América Latina. México: Fondo de Cultura Económica, 2006. p. 15-99

DURKHEIM, Emile. La división del trabajo social. Madrid: Ediciones Akal, 1987.

EDWARDS, Bob; FOLEY, Michael. Civil society and social capital: A primer. In: EDWARDS, Bob; FOLEY, Michael; DIANI, Mario (Ed.). Beyond Tocqueville: civil society and the social capital debate in comparative perspective. New England: University Press of New England, 2001. p. 1-14.

FINE, Ben. The developmental state is dead - long live social capital? Development and Change, v.30, n. 1, p. 1-19, 1999.

FISHMAN, Robert. Why I don't particularly like the concept of social capital: Notes on a contemporary social science fashion. Barcelona: Universitat Pompeu Fabra, 2000. (Inédito)

GARCÍA INDA, Andrés. Introducción: la razón del derecho: entre habitus y campo. In: BOURDIEU, Pierre. Poder, derecho y clases sociales. Bilbao: Desclée de Brouwer, 2001. p. 9-60.

GERRING, John. What makes a concept good? A criterial framework for understanding concept formation in the social sciences. Polity, v. 31, n. 3, p. 357-393, Spring 1999.

HALL, Peter. Social capital in Britain. British Journal of Political Science, v. 29, n. 3, p. 417-461, 1999.

JACKMAN, Robert; MILLER, Ross. A renaissance of political culture? American Journal of Political Science, v. 40, n. 3, p. 632-659, 1996.

JORDANA, Jacint. Instituciones y capital social: ¿qué explica qué? Revista Española de Ciencia Política, v. 1, n. 2, p. 187-210, 2000.

LEVI, Margaret. Social and unsocial capital: A review essay of Robert Putnam's Making Democracy Work. Politics \& Society, v. 24, n. 1, p. 45-55, 1996. 
LIN, Nan. Social capital: a theory of social structure and action. Nueva York: Cambridge University Press, 2001.

LOURY, Glenn. A dynamic theory of racial income differences. In: WALLACE, Phyllis; LAMOND, Annette. Women, Minorities, and Employment Discrimination. Lexington, USA: Lexington Book, 1977. p. 86-153.

MACEDO CASTILLEJOS, Ignacio. Two Decades of Social Capital: Where is this Concept Going? The International Journal of Interdisciplinary Social Sciences, v.4, n.1, p. 243-257, 2009.

PORTES, Alejandro. Social Capital: its origins and applications in Modern Sociology. Annual Reviews Sociological, v. 24, p. 1-24, 1998.

. Social capital: Its origins and applications in modern sociology. In: LESSER, Eric L. Knowledge and Social Capital. Boston: Butterworth-Heinemann, 2000. p. 43-67.

PUTNAM, Robert. Making democracy work: Civic traditions in modern Italy. Princeton: Princeton University Press, 1993.

Bowling alone: the collapse and revival of American community. New York: Simon and Schuster, 2000.

RUBIO, Mauricio. Perverse social capital: some evidence from Colombia. Journal of economic issues, p. 805-816, 1997.

SCHNEIDER, Cecilia; AVENBURG, Karen. Cultura política: un concepto atravesado por dos enfoques. Revista Postdata, v. 20, n. 1, abr.-set. 2015.

SIMMEL, George. Sociología: estudios sobre las formas de socialización. Madrid: Alianza Editorial, 1986.

SUDARSKY, John. Colombia's social capital: the national measurement with the BARCAS. World Bank, Washington, p. 1-48, 1999.

TZANAKIS, Michael. Social capital in Bourdieu, Coleman and Putnam's theory: empirical evidence and emergent measurement issues. Educate, Londres, v.13, n. 2, p. 2-23, 2013. Disponível em: <http://www.educatejournal.org/index.php/educate/article/view/366>. Acesso em: 07 abr. 2015.

WORLD BANK. Measurement Tools. Disponível em: <http://go.worldbank.org/KO0QFVW770>. Acesso em: 17 abr. 2015.

Texto recebido em 17 de abril de 2015 . Aprovado em 04 de agosto de 2015. 
